\title{
Correction to: Convergence Analysis of a Crank-Nicolson Galerkin Method for an Inverse Source Problem for Parabolic Equations with Boundary Observations
}

\section{Dinh Nho Hào ${ }^{1} \cdot \operatorname{Tran}_{\text {Nhan Tam Quyen }}^{2}$ (D) Nguyen Thanh Son ${ }^{3,4}$}

Published online: 14 September 2020

(c) Springer Science+Business Media, LLC, part of Springer Nature 2020

\section{Correction to:}

\section{Applied Mathematics \& Optimization} https://doi.org/10.1007/s00245-020-09710-2

The original version of this article unfortunately contained a mistake in one of the co-author's name. A part of the coauthor given name Tran Nhan Tam's was missing. The correct name is Tran Nhan Tam Quyen.

The original article has been corrected.

Publisher's Note Springer Nature remains neutral with regard to jurisdictional claims in published maps and institutional affiliations.

The original version of this article was revised: the missing given name of Tran Nhan Tam Quyen was added.

The original article can be found online at https://doi.org/10.1007/s00245-020-09710-2.

Tran Nhan Tam Quyen

quyen.tran@uni-goettingen.de

Dinh Nho Hào

hao@math.ac.vn

Nguyen Thanh Son

thanh.son.nguyen@uclouvain.be

1 Hanoi Institute of Mathematics, VAST, 18 Hoang Quoc Viet Road, Hanoi, Vietnam

2 Institute for Numerical and Applied Mathematics, University of Goettingen, Lotzestr. 16-18, 37083 Goettingen, Germany

3 ICTEAM Institute, Université catholique de Louvain, 1348 Louvain-la-Neuve, Belgium

4 Department of Mathematics and Informatics, Thai Nguyen University of Sciences, Thai Nguyen 25000, Vietnam 